\title{
Chaos and complexity in astrophysics
}

\author{
Oded Regev ${ }^{1,2}$ \\ ${ }^{1}$ Department of Physics, Technion-Israel Institute of Technology, Haifa, Israel \\ 2 Department of Astronomy, Columbia University, New York, NY 10027, USA
}

\begin{abstract}
Methods and techniques of the theory of nonlinear dynamical systems and patterns can be useful in astrophysical applications. Some works on the subjects of dynamical astronomy, stellar pulsation and variability, as well as spatial complexity in extended systems, in which such approaches have already been utilized, are reviewed. Prospects for future directions in applications of this kind are outlined.
\end{abstract}

to appear in the

Springer Encyclopedia of Complexity and System Science

http://refworks.springer.com/complexity 


\section{APOLOGIES AND EXPLANATIONS}

I apologize for not being able to provide the readers with the pleasure of downloading this preprint directly from the arXiv: astro-ph archive. Intensive efforts on my part to post this preprint on the arXiv:astro-ph have unfortunately failed. It is undoubtedly due to my ignorance in the advanced methods of computer science, required for this task. I have posted papers before on the preprint archive, but this time my .eps figure files were either "too large" or "not sufficiently portable" so that the posting was repeatedly rejected despite my heroic efforts to improve the "encoding", "compression" etc. of the miserable .eps files.

Neither Cambridge University Press (CUP) nor Springer-Verlag (SV) had any problems with the same figure files. They were happily accepted and were either already published (in my CUP book) or will be published in the future (in the SV Encyclopedia). My old laptop also easily compiled the LaTeX file, embedded these figures and produced a .pdf file of $\sim 2.1 \mathrm{MB}$ (obviously prohibitively large for a mere 30 page article, with 11 figures, and reflecting the author's incompressibility).

Interested readers are invited to download the manuscript in .pfd (with the figures!) from my home-page, residing on the disk of a very modest computer at my Department: http://physics.technion.ac.il/ regev/ccastro.pdf [1] I hope that a direct download of a .pdf file, without it being created first by the sophisticated software and hardware of the arXiv, will be sufficiently adequate for most purposes.

\section{ACKNOWLEDGEMENT}

I am grateful to all those colleagues of mine (too numerous to be all mentioned here, lest the file be too large) who tried, unsuccessfully, to help me in this endeavor.

This work was not supported by any grant and mere words of encouragement to regev@physics.technion.ac.il will be highly appreciated.

[1] The links in this document are non-clickable. Please re-type them in your browser. Sorry, I can't figure out how to type the "tilde" character as text in TeX. 